\title{
Fishing Boat of the Waveless Hull Form
}

\author{
By Nobutatsu Yokoyama, * Member.
}

\section{Summary}

This paper describes an experimental survey of the bulbous bow effect on the resistance with fishing boat models of existing design and a comparative investigation between theoretical aspects based on the waveless hull form theory ignited by Prof. INUI') and experimental results with a calculated model of known sink-source distribution; and after advocating the necessity of an original development of unprecedented hull form for realizing actually waveless state, the paper gives some tank test results of self-propulsion and of seakindliness among waves with a model of exploratory design for practical waveless boats. These preferable results may stimulate the further persuit of another effective hull form for the bulb not protruding from the bow so as not to prevent from easy operation of anchoring and steering.

\section{Introduction}

There would be found some apparent differences as shown in Table 1 and Fig. 1 in the proportion of speed, displacement and power to the ship length between fishing boats in Japan smaller than $70 \mathrm{~m}$. and average larger cargo liners. These statistical results since 1950 may be interpreted as there have been no other ways than powering up engine and any hydrodynamical efforts have not been paid to impiove hull shape in preventing the expected speed loss resulted from the increase of ship-displacement by swelling out $C_{b}$ to leave $L$ unchanged on acount of the ruled restriction for LBD or GT. Conseqently the effect of full $C_{b}$ on the wave resistance must of necesity appear on the results of tank test in Fig. 2 which has been conducted by the Fishing Boat Laboratory, Fisheries Agency, with the Japanese fishing boat models built for these ten years. Considering that in Fig. 1 most operating speed at sea comes between $V / \sqrt{L g}=0.25$ and 0.30 it is evident in Fig. 2 that most full ships have a peculiar fatality of climing up the confronted hump of the wave resistance and, that is, they have to waste excess power of some extent which might be saved by a hydrodynamical improvement for the hull form. There may possibly be two methods of the improvement; viz. the one of reducing wave resi-

Table I Ratios for general boats

\begin{tabular}{|c|c|c|c|c|c|c|}
\hline & or & $L / B$ & $\left.\nabla^{8} / 12\right)^{3}$ & $\nabla / \sqrt{L_{8}}$ & $\mathrm{EP} / \mathrm{GP}$ & $\boldsymbol{s}_{\mathrm{b}}$ \\
\hline passenger boat & 126 & 4.17 & 9.0 & .362 & 2.54 & .521 \\
\hline cham1cal tanker & 146 & 4.66 & 13.1 & .252 & 0.82 & .705 \\
\hline oil tanker & 199 & 5.59 & 11.2 & .278 & 1.41 & .771 \\
\hline do. & 290 & 5.52 & 11.4 & .268 & 1.38 & .740 \\
\hline cargo boat & 499 & 5.70 & 9.9 & .270 & 1.60 & .722 \\
\hline fish oarrier & 1391 & 6.35 & 7.7 & .250 & 1.12 & .687 \\
\hline 011 tanker & 2262 & 6.44 & 8.0 & .187 & 0.88 & .753 \\
\hline forr, boat & 4982 & 6.37 & 7.1 & .203 & 0.67 & .772 \\
\hline cargo boat & 9548 & 7.41 & 5.7 & .235 & 1.18 & .699 \\
\hline do. & 14302 & 7.39 & 5.9 & .203 & 0.84 & .730 \\
\hline 011 tanker & 28972 & 6.99 & 6.2 & .193 & 0.61 & .809 \\
\hline do. & 31109 & 6.69 & 6.4 & .180 & 0.51 & .809 \\
\hline
\end{tabular}

CT: grose tonrage, L/Biratio of $L_{p p}$ to molded breadth, $\nabla_{0}$ i displaconent

of fully loadod snip, La pp: length botween porpandiculars, $v^{*}$ : servico

s coed, BP:rated horse power, Cb:block coeffiaiont for full-load coption 

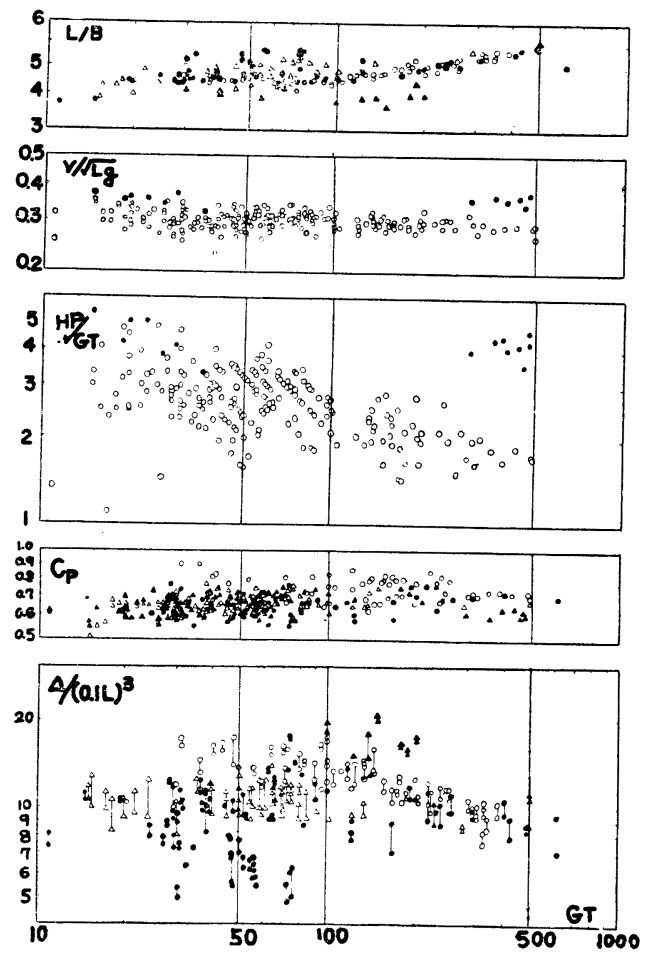

Fig. 1 Ratios for fishing boots; fisheries, inspection boat, boni-tuna long line boat, $\Delta$ trawler, $\Delta$ the other fishing boats.

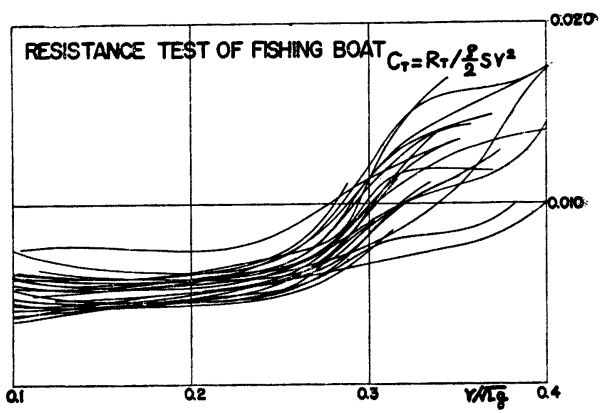

Fig. 2

stance with a hull-shape transformation or of an escape from making wave by submerging under water or being dynamically lifted above; and the other of actively cancelling the formation of ship wave by a counteracting body specially designed on the "waveless-hull form theory."

The fascinating theory has been established by Prof. INUI (1960) ${ }^{1}$ ) based on his exact calculation of wave-making resistance and carefull analysis of the experiment, and it has predicted possibility of the exsistence of a real waveless-hull form. For the bow waves and the stern waves respectively TAKAHEI $^{2)}$ and $\mathrm{KUMANO}^{3)}$ (1960)

have proved the precise coincidence between the theory and the experiment conducted with two theoretically calculated models. And in 1961 an actual sea test was undertaken by these investigators with $80 \mathrm{~m}$. passenger boat, "KURENAI MARU" and its waveless aspect was successfully filmed from a helicopter. In applying this fundamental theorem, however, to practical fishing boat design not only some more details should be explored, but the substantial difference should also be recognized from the existing design of bulbous bow. The author points out in this paper an important recognition that the bulb would not always become a means of reducing the wave-making resistance of any kind of ships but only when combinded with a specific form of hull which should be designed on an entirely new stand-point it will work as an effective wave erasor at an intended speed.

The present paper gives first exemplified experiments with models of existing fishing boats either on the usual bulbous bow design or on the "waveless theory" and shows the former a chance example of increasing resistance and the latter in somewhat effective but imcomplete waveless condition. A close analysis of the waves generated from every part of the existing hull proves the situation resulted from unfavourable wave component for the counteraction. The author contributed to give somie information of potential use to practical design on thorough investigations about the wave counteraction with a mathematical model, the proportion of whose dimension is quite similar to the average fishing boat, and presented another favourable test results for a waveless trawler model of new fashion by making the best possible use of these results.

\section{Bulb for existing boats}

Generally the ship waves are composed of the local disturbances at bow and stern, and the free waves discharged behind the ship. The former is quite free from the wave resistance since they are 
longitudinally symmetrical in ideal fluid, but the latter has much the most complicated effect on it. The elevation of the free waves $\zeta_{w s}$ of ships for practical use is expressed in general form as :

$$
\zeta_{w s} \sim \sum_{i} \int_{-\pi / 2}^{\pi / 2} A_{i}\left(V, m_{i}, T_{i}, \theta\right) \sin \left(V, x+\delta_{i}, y, \theta\right) d \theta
$$

In case that the ship is possibly represented by an equivalent source distribution $m(\xi, \zeta)$, the above amplitude function $A_{i}$ in (1) may become

$$
A_{i}=\frac{g \sec ^{3} \theta}{\pi V^{3}} \int_{-T_{i}}^{0} \exp \left(\frac{g}{V^{2}} \zeta \sec ^{2} \theta\right) \int_{\xi_{i}} m_{i}(\xi, \zeta) d \xi d \eta
$$

Similarly the free wave elevation of the bulb may be expressed as following :

$$
\zeta_{w B} \sim \int_{-\pi / 2}^{\pi / 2} B(V, M, f, \theta) \sin (V, x+\varepsilon, y, \theta) d \theta
$$

The wave amplitude function of counteracted wave $(C)$ and the transfer of the resultant profile $(\delta)$ are consequently obtained as

$$
\left.\begin{array}{c}
C^{2}=\left[\sum_{i}\left(A_{i} \cos \delta_{i}^{\prime}\right)+B \cos \varepsilon^{\prime}\right]^{2}+\left[\sum_{i}\left(A_{i} \sin \delta_{i^{\prime}}\right)+B \sin \varepsilon^{\prime}\right]^{2} \\
\delta^{\prime}=\tan ^{-1}\left[\left\{\sum\left(A_{i} \sin \delta_{i}^{\prime}\right)+B \sin \varepsilon^{\prime}\right\} /\left\{\sum\left(A_{i} \cos \delta_{i}^{\prime}\right)+B \cos \varepsilon^{\prime}\right\}\right]
\end{array}\right\}
$$

where $V$ denotes ship speed; $m_{\imath} \& M$ : strength of equivalent source and doublet; $T_{i} \& f:$ draft and depth of $m_{i}$ and $M ;(x, y, \theta):$ coordinate of a considering point in the fluid surface and direction from $m_{\imath}$ or $M ; A_{i} \& B$ : amplitude function of ship waves and bulb. Then the resulting wave resistance, therefore, will be represented by

$$
R_{w}=\pi \rho V^{2} \int_{0}^{\pi / 2} C^{2} \cos ^{3} \theta d \theta
$$

When the stern wave can be put aside, the condition necessary for the least wave resistance is obviously $C \rightarrow$ min., and this will be realized when

$$
\left.\begin{array}{l}
B=-A_{\text {bow }}, \quad \delta_{\text {bow }}=\varepsilon \\
A_{\text {i except bow }} \rightarrow 0
\end{array}\right\}
$$

The composition of the summation $\sum_{i}$ in (1) or the compornent amplitude $A_{i}$ in (2) has much important influence on the effectiveness of waveless counteraction as shown explicit in the following experiments (a) and (b), and it entirely depends upon the shape of ship's lines_-not only the coefficients, $C_{p}, C_{m}, C_{w}$, but also the form of waterlines and bow-buttock lines, even if the case of having the same coefficients.

(a) Ineffective example : A bulbous bow model, M-575 BB, (CF : Table II \& III) of usual design heretofore in use was prepared by means of being transformed from the ordinary model, M-575, by shifting $10 \%$ of the original ship's volume to the bulb inside the stem to keep the displacement unchanged, which has low prismatic coefficient. 0.575 and gives excellently low resistance between $V / \sqrt{L g}=0.25$,

\begin{tabular}{|c|c|c|c|c|c|c|}
\hline & \multicolumn{4}{|c|}{ morinl of exteting amall tramler } & \multicolumn{2}{|c|}{$\begin{array}{l}\text { model of } \\
\text { vavoloss hull }\end{array}$} \\
\hline & \multicolumn{2}{|c|}{$\begin{array}{c}\text { (Buropoan) } \\
M-575 \quad M-675\end{array}$} & \multicolumn{2}{|c|}{ 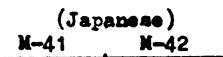 } & $x-43$ & $x-44$ \\
\hline$L_{p p}(m)$ & 2.000 & 2.000 & 1.963 & 1.963 & 2.000 & 2.000 \\
\hline Bnold (m) & 0.544 & 0.502 & 0.388 & 0.424 & 0.410 & 0.410 \\
\hline Tpore (m) & 0.160 & 0.145 & 0.142 & 0.155 & 0.100 & 0.100 \\
\hline (m) & 0.200 & 0.185 & 0.168 & 0.183 & 0.267 & 0.240 \\
\hline$B / L$ & 0.272 & 0.251 & 0.198 & 0.215 & 0.205 & 0.205 \\
\hline$T_{f} / \mathrm{J}$ & 0.0800 & 0.0725 & 0.0724 & 0.0790 & 0.0500 & 0.0500 \\
\hline $\mathrm{Ta} / \mathrm{L}$ & 0.1000 & 0.0825 & 0.0856 & 0.0932 & 0.1335 & $0: 1200$ \\
\hline$\nabla \quad\left(m^{3}\right)$ & 0.09920 & 0.09920 & 0.07663 & 0.07663 & 0.10867 & 0.10773 \\
\hline$\left(m^{2}\right)$ & 1.2364 & 1.1904 & 1.0806 & 1.1466 & 1.1337 & 1.3150 \\
\hline An $\left(m^{2}\right)$ & 0.0863 & 0.0735 & 0.0603 & 0.0697 & 0.0841 & 0.0818 \\
\hline$\nabla /(0.1 L)^{3}$ & 12.40 & 12.40 & 10.13 & 10.13 & 13.58 & 13.47 \\
\hline$\Delta-/ L T_{p}$ & 0.270 & 0.253 & 0.216 & 0.229 & 0.421 & 0.409 \\
\hline
\end{tabular}
and 0.30 according to the comparative resistance test programmed by TRAUNG ${ }^{(4)}$ (1957), FAO, UN, and carried out by the author ${ }^{(5)}(1958)$. Contrary to the initial anticipation of reducing resistance the towing

Table II. Particulars of the models feos aren, ins midehip neotion area. 
Table III Particulars of the Bulbs

\begin{tabular}{|c|c|c|c|c|c|c|}
\hline & $\Delta \nabla\left(n^{3}\right)$ & $\Delta s \quad\left(a^{2}\right)$ & $\Delta b / d e$ & $a_{0} / L$ & $\mathrm{~b} / \mathrm{L}$ & P/L \\
\hline $\mathrm{BB}(u-575)$ & 0 & 0.0044 & 0.100 & 0.020 & -0.025 & 0.055 \\
\hline BB $(M-675)$ & 0.00393 & 0.0905 & $0.209_{3}$ & 0.035 & 0.015 & 0.0445 \\
\hline BB $(x-41)$ & 0.00307 & 0.1007 & 0.2904 & 0.038 & 0.038 & 0.0475 \\
\hline $\begin{array}{l}B B(M-42) \\
B B 1(x-43) \\
B B 2(\text { do. })\end{array}$ & $\begin{array}{c}0.00228 \\
0.00485- \\
0.01207 \\
0.00452\end{array}$ & $\begin{array}{c}0.0596 \\
0.0994- \\
0.2443 \\
0.1181\end{array}$ & $\begin{array}{l}0.122 \\
0.3735 \\
0.269 q\end{array}$ & $\begin{array}{l}0.025 \\
0.050 \\
0.0425\end{array}$ & $\begin{array}{c}0.0235 \\
0.010- \\
0.125 \\
0.0425\end{array}$ & $\begin{array}{c}0.052 \\
0.050- \\
0.125 \\
0.075\end{array}$ \\
\hline BB3 (do.) & 0.00275 & 0.0936 & 0.1937 & 0.036 & 0.036 & 0.075 \\
\hline BB $(x-44)$ & 0.00736 & 0.1609 & 0.1715 & 0.037 & $0.037 \mathrm{~s}$ & 0.0825 \\
\hline
\end{tabular}

$B B:$ bow bulb, $\Delta \nabla_{2}$ volume of builb, $\Delta A_{1}$ surfaco aroa of bulb, $A_{b}: \max$. sectional area of bulb, Ans midship area, a : radius of estimated bulb sphere, bi distance of bulb center from FP, (forward possitivo), Ps ismersion of bulb center, L: length betwoen porpendiculare. test with the model showed a noticeable increase, for instance shown in Fig. 3. By an analysis of shipside profiles of the resultant wave like an example in Fig. 4, it is clearly realised that the fact was introduced by wrong counteraction between the bow wave and the relatively mislocated bulb. Besides this model a large extruded bulb was fitted for a comparison outside the stem of M-575 and M-42 keeping their draft as same as the ori-

ginal, both of which are much the finer models (CF : Table II \& III) $-C_{p}$ of the latter is likewise 0.580 - than the ordinarily built nowadays and showed good results in towing and also in self-propel-

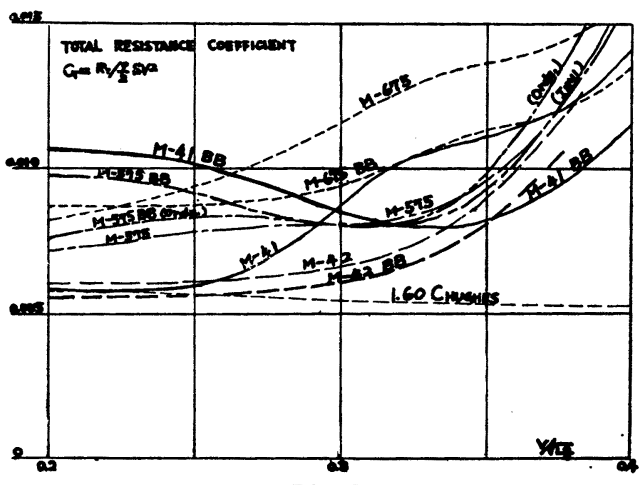

Fig. 3

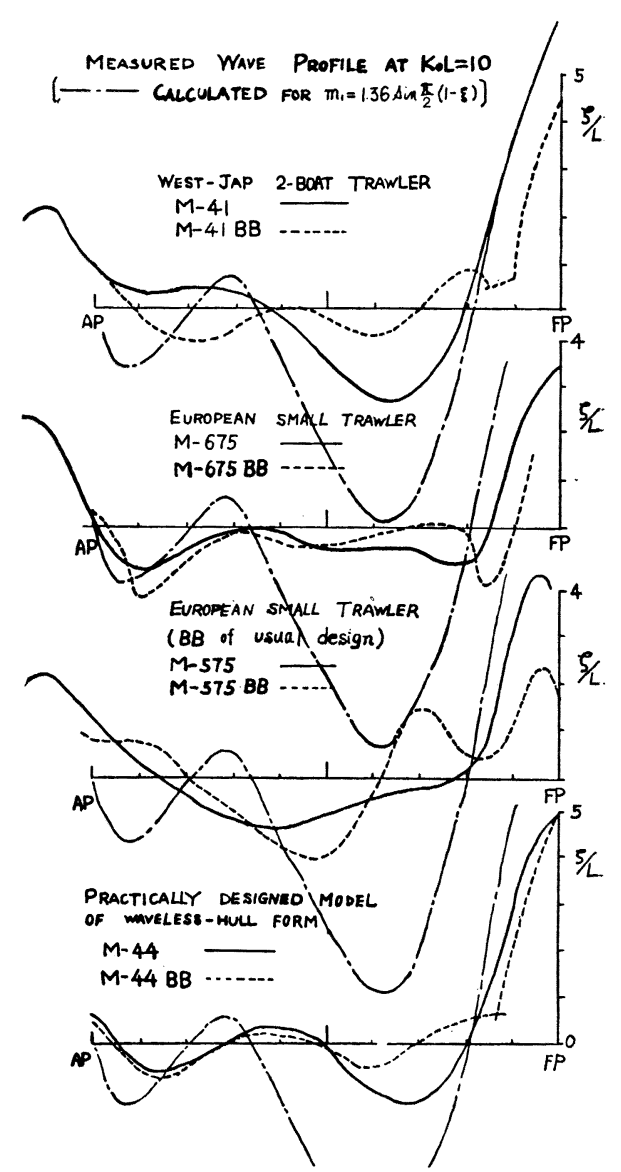

Fig. 4

led test as mentioned before. The towing results in

Fig. 3, however, are not so appreciable as expected.

Such ineffectiveness as those must be brought by some influential wave components of parasitic sources distributed in both hulls which have been modified forced to have quite low prismatic.

(b) Incomplete example : For the study of further effect on existing fishing boats of full fore- and aftbody, M-675 and M-41 (CF : Table II \& III) whose $C_{p}$ are 0.675 and 0.633 are submitted to the test coupled with a phase-adjusted bulb after the ship-side wave examined. The test result in Fig. 3 indicates a preferable reduction at $V / \sqrt{\mathrm{Lg}}=0.35$ and that may be resulted in on account of the cancellation of the bow wave which represents the major component of the whole wave generated by the rather simple-formed hull.

The more carefully, however, the water surface is observed, still the more unoverlooked wave could: be found remain and not partially cancelled wave is left. This phenomenon may be comprehended"to. depend upon its incomplete waveless form of main hull. 
The facts, (a) and (b), instruct us nothing but the importance of the developement for a peculiar hull not having parasitic sources and of the investigation of the effect of bulb location.

\section{The eff ect of bulb}

A comprehensible frame-work for the effect of bulb on the resultant wave resistance could be given by the following ratio of amplitude function,

$$
\left(\frac{C}{A}\right)^{2}=\left(1-\frac{B}{A}\right)^{2}+2\left[1-\cos \left(K_{0} b \sec \theta\right)\right] \frac{B}{A}
$$

where $b$ means the distance between each location of the wave source for $A$ and $B$. The effect of bulb size and locations will be analogized by the illustration of Fig. 5 in the form of parameters, $\left(a_{0} / L\right)^{3} / a_{1}$, $f / L, \cos \left(K_{0} b\right)$, when $\theta=0$ for the simplification, where $K_{0}=g / V^{2}$ and $a_{0}$ means the radius of the spherical bulb. The truer resistance may be expressed by superposing on these curves the other effects of $\theta$, fundamental terms of the other component waves and one another's interfering terms which should disappear right at the complete waveless points generally found at certain two Froude numbers. From the above illustration it would be understood that the complete waveless state could be realized only when $b$ $=0$ and in the incomplete counteraction there might be even a case of resistance increasing.

These caluculated aspects are precisely proved in Fig. $6,7,8$ by the experiments with a mathematical model M-43 whose lines are obtained along the streamlines of flow produced by the distributed source and sink $V \cdot m(\xi, \zeta)$ as follows in the uniform stream $V$ without free surface :

$$
\begin{aligned}
m(\xi, \zeta)= & m_{1}(\xi) \cdot m_{2}(\zeta) ;-1 \leqslant \xi \leqslant 1 ;-t \leqslant \zeta \leqslant t \\
& m_{1}(\xi)=a_{1} \sin (\pi \xi / 2) ; a_{1}=1.36
\end{aligned}
$$
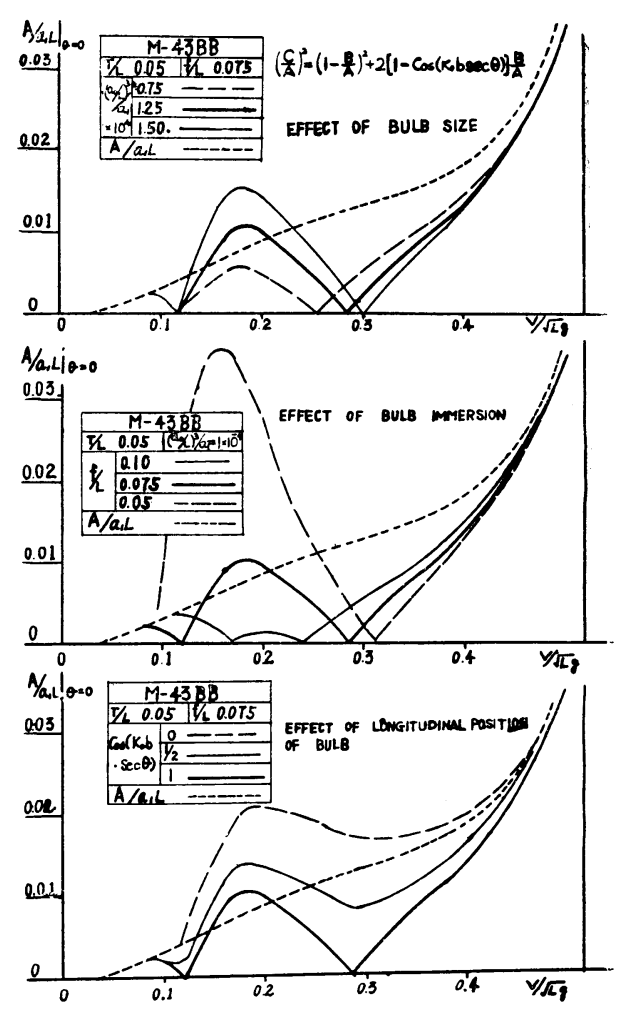

Fig. 5

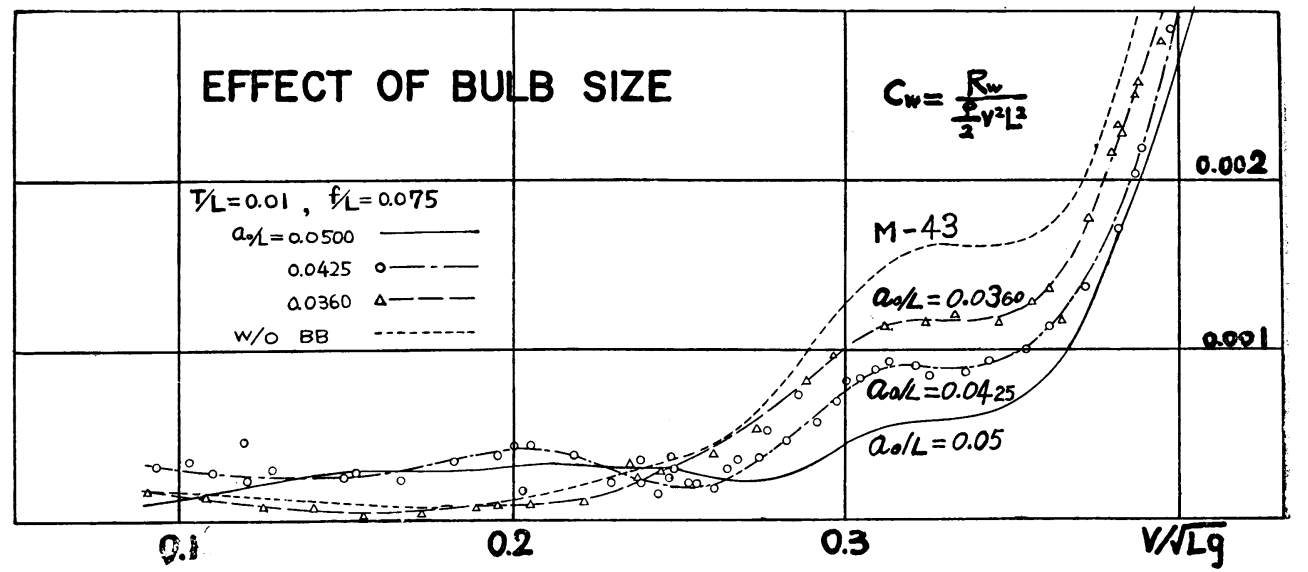

Fig. 6 


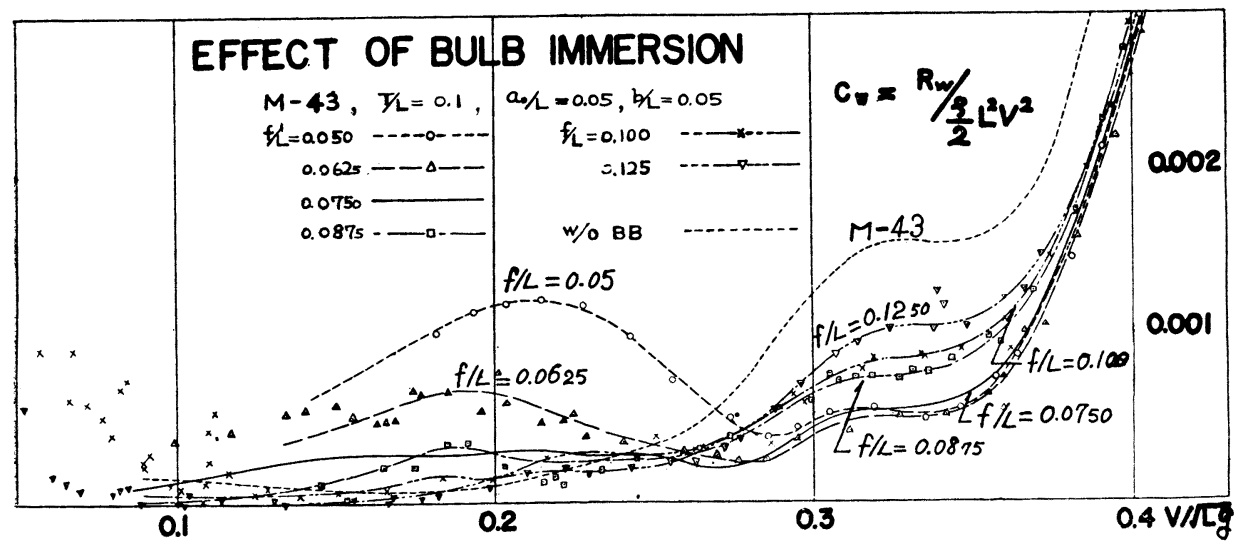

Fig. 7

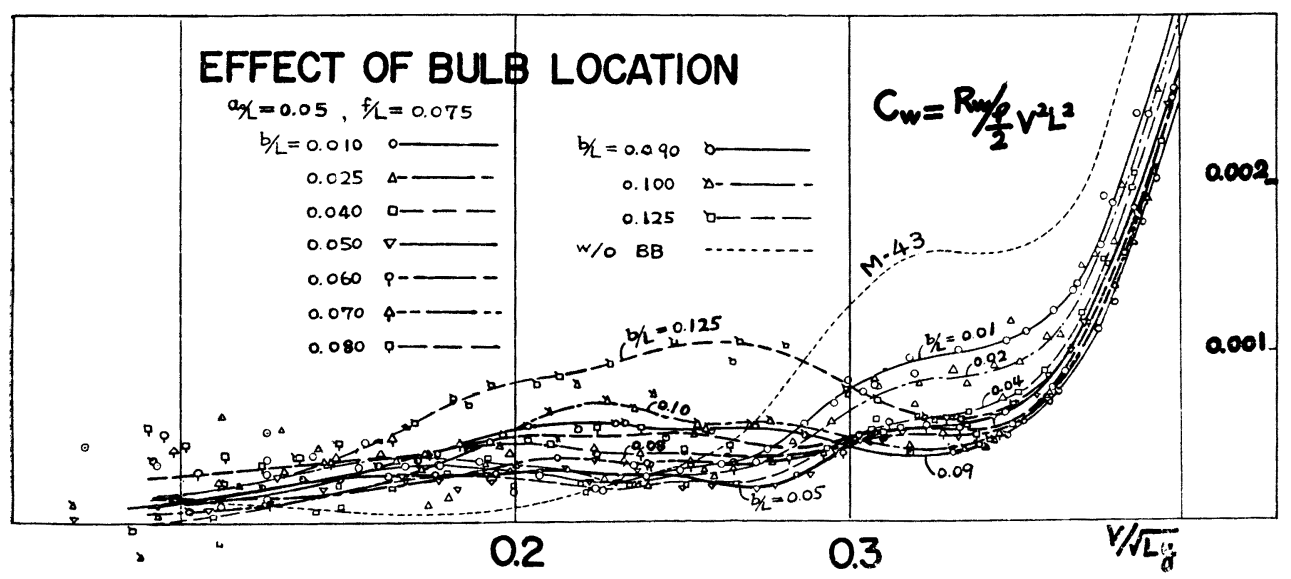

Fig. 8

$$
m_{2}(\zeta)=1 \quad ; t=0.05
$$

and the dimension of $\mathrm{M}-43$ is given in Table II \& III. In the above experiment the wave resistances are deduced by reducing the form drag from the resultant according to the Hughes method assuming form factor as common as $k=0.60$, because of the indeterminate phase of the total resistance at low speed on account of the interaction. The turbulence of the model surface is stimulated by a row of plate studs placed on the station of 9.5 and the turbulent separation is maintained by stimulators of the same kind arranged on the bulb at $a_{0} / 2$ abaft the front end.

The calculation of the free wave produced by the source distribution (8) shows that the bow and stern wave should be created exactly at FP. and AP. In reality, huwever, the distribution for M-43 running on the water surface should be modified in the approximation of second order at best to satisfy the condition of ship surface especially under the perturbation of free wave, and in consequence the generating point of bow or stern wave may possibly be shifted by the modification as far as the following distance afore FP. or abaft AP. assuming $F^{n} \rightarrow 0$ when $n \geqslant 6$ :

$$
\frac{b}{L} \sim \frac{1}{K_{0} L} \tan ^{-1}\left(-\frac{\partial \zeta}{\partial x}\right)_{x=0} K_{0} L=1 / F^{2}
$$

where $b=$ shift of the wave profile, $\zeta=$ elevation of the wave surface.

Such tendency as the above mentioned is also confirmed by the experiment, in which the center of bulb comes to project as far as $5 \%$ of $L$ from FP. at a waveless point, $V / \sqrt{L g}=0.270$. 
The size of the bulb should be decided both by the amplitude function of the main hull and of the bulb in (3), where the latter could be practically replaced by a doublet, especially in spherical bulb. For the model M-43 with $U$-frame line

$$
\left.\frac{A}{L}\right|_{U} \sim \frac{a_{1}}{\pi} K_{0} L \frac{\left[1-\exp \left(-K_{0} T \sec ^{2} \theta\right)\right] \sec ^{2} \theta}{\left(K_{0} L \sec \theta\right)^{2}-\pi^{2}}
$$

and samely for the $V$-frame model, $m_{2}(\zeta)=1+\zeta / t$

$$
\left.\frac{A}{L}\right|_{V} \sim \frac{a_{1}}{\pi} \frac{L}{T} \frac{K_{0} T \sec ^{2} \theta-1+\exp \left(-K_{0} T \sec ^{2} \theta\right)}{\left(K_{0} L \sec \theta\right)^{2}-\pi^{2}}
$$

These could be generalized in the following form applying the Michell's assumption for thin ships$u \frac{\partial y}{\partial x}=w \frac{\partial y}{\partial z} \rightarrow 0, v=-V \frac{\partial y}{\partial z}$ to the fore end of the model $a_{1} \sim 2 \alpha$, where $V \cdot m$ means flow volume per unit time and area; $\alpha$ half angle at the entrance of water-line $;(u, v, w)$ a flow velocity $(x, y, z)$ a point on the surface of ship; $V$ the ship speed; $F=V / \sqrt{L g}=1 / \sqrt{ } K_{0} L$ :

$$
A / L \sim(2 \alpha / \pi) \boldsymbol{F}(F, T / L, \theta)
$$

And the amplitude function of a spherical bulb, the draft of whose center is $\zeta=-f$,

$$
\frac{B}{L} \sim-2\left(\frac{a_{0}}{L}\right)^{3}\left(K_{0} L\right)^{2} \sec ^{4} \theta \exp \left(-K_{0} f \sec ^{2} \theta\right)
$$

in general

$$
\sim-\left(\frac{a_{0}}{L}\right)^{3} G(F, f / L, \theta)
$$

Since at complete waveless point $B / A=1$ and $b=0$ in $(7)$,

$$
B_{*} \equiv \frac{\left(a_{0} / L\right)^{3}}{2 \alpha / \pi}=\frac{\boldsymbol{F}(F, T / L, \theta)}{\boldsymbol{G}(F, f / L, \theta)}
$$

Fig. 9 is the example of $B_{*} / \pi$ for M-43 of $U$-frame, to which (10) and (12) are applicable. From the illustration at $F=0 \cdot 275, f / L=0 \cdot 100$

$$
a_{0} / L=\sqrt[3]{2 \alpha B_{*}} / \pi=0.05 \text { for } a_{1}=1.36
$$

and fortunately this coincides with the experiment.

\section{Waveless trawler model for practical use}

Through the foregoing investigation it would

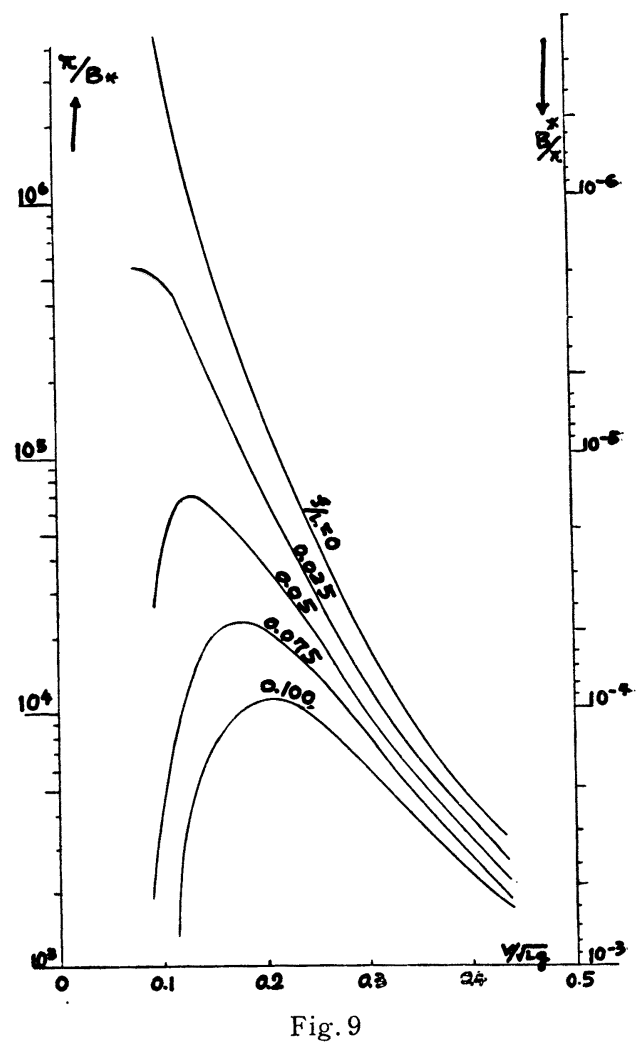
be recognized that the effectiveness of the bulb at a designed speed depends upon the lines of the main hull having no parasitic wave except the bow and stern (paragraph 2) and upon the size and location of the bulb relative to the bow or stern wave(paragraph 3 ). The former hull form could be obtained

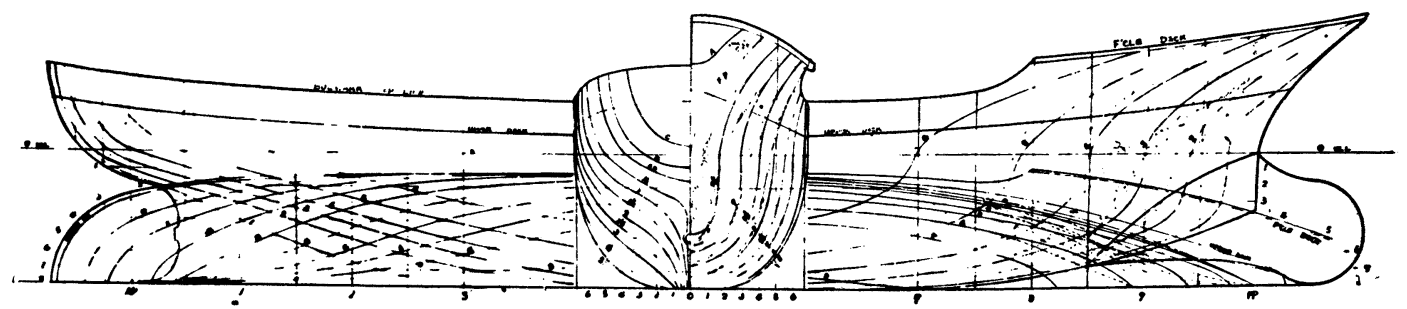

Fig. 10 
by the similar theoretical calculation for $\mathrm{M}-43$ for example and the latter could be estimated by the experimental analysis of wave profile and the theoretical deliberation (paragraph 3). Under these considerations a practical trawler model, M-44 BB (CF : Table II \& III), was derived after some complementary exploring with M-43 about an effect of flat bottom and of finer entrance etc. The forebody of M-44 in Fig. 10 is almost the same as M-43 except a slightly flat bottom and a little finer entrance, and the aft-body is lifted flat upwards to have satisfactory reserve buoyancy for trawling and to avoid the separation of flow. The point a full deliberation should be placed on is that these

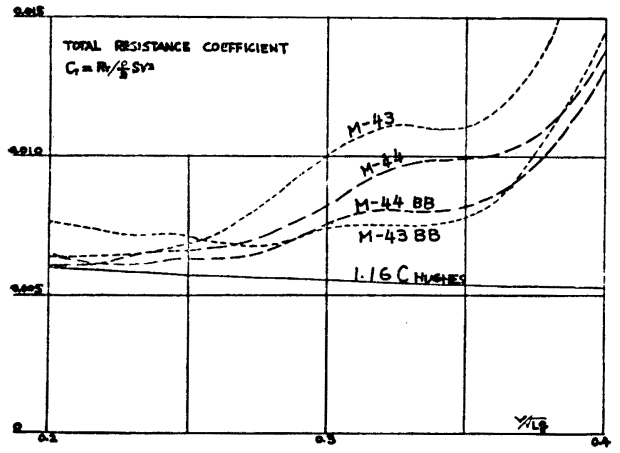

Fig. 11 transformation for practical boat has to be tried to maintain the characteristics of bow wave of $M$ -43 as well as possible which is effectively able to be cancelled with a bow-bulb and not to increase the stern and the other parasitic waves. As shown by the result in Fig. 11, M-44 BB would possibly be regarded as a fairly successfull practical model of waveless hull form counting on its considerable reduction of resistance. If allowed to hope for more, the bulb should be made somewhat larger to meet a little higher waveless speed and located a little forward to complete waveless effect if not work against practical manoeuvring operation or, otherwise, a more hollow fore-body should be investigated.

Propulsive factors are compared between the self-propulsion tests with 2-m. model of M-44 and M $-44 \mathrm{BB}$ fitted with the stock propeller of $0.145 \mathrm{~m}$. in diameter and $0.087 \mathrm{~m}$. in pitch. Accrding to the result in Table IV $10 \%$ gain in hull efficiency at $V / \sqrt{L g}=0.30$ is brought about by $7 \%$ increase of wake which may be resulted in on account of the diminution of negative wave wake.

Table IV.

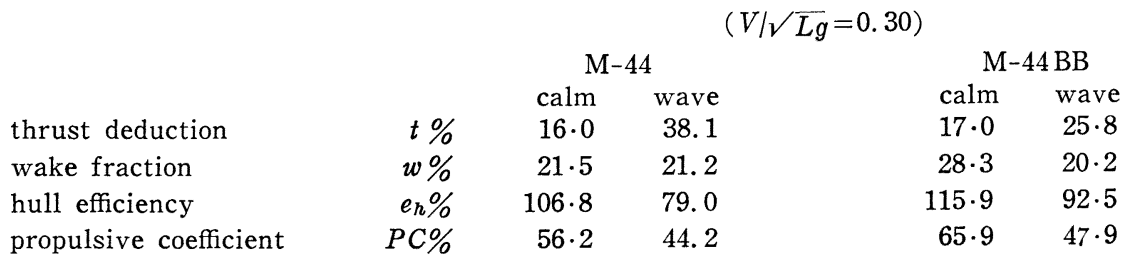

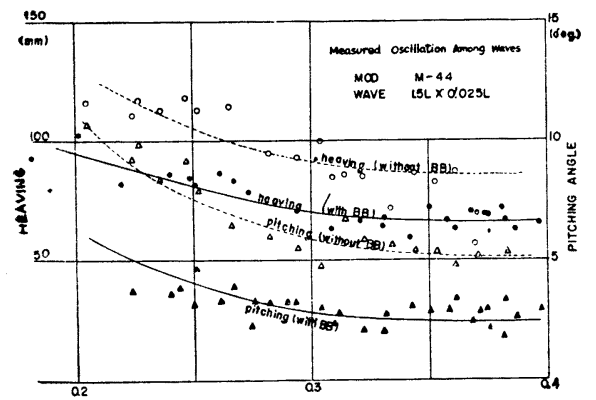

Fig. 12

A comparative wave test with the same models having $0.25 \mathrm{~L}$ radius of gyration is carried out in the wave of $1.5 \mathrm{~L}$ long and $\mathrm{L} / 40$ high and the period of free pitching is $1.17 \mathrm{sec}$. for $\mathrm{M}-44$ and $1.27 \mathrm{sec}$. for $\mathrm{M}-44 \mathrm{BB} .4 \%$ gain in the thrust deduction for $\mathrm{M}-44$ $\mathrm{BB}$ may come from a reduction of motion by the restricting effect of bulb as shown later in Fig. 12. The cause of the equivalent PC for both models contrary to the considerable difference in hull efficiency lies in the fall of relative rotative efficiency for M-44 $\mathrm{BB}$ which is inflicted by an increase of relative motion between wave and the model as a result of the restricting effect by the bulb.

The motion among waves is considerably holded by the bulb as recorded in Fig. 12 and Fig. 13. The pitching amplitude is reduced to half of that of the model without the bulb and the heaving amplitude 
is improved to $80 \%$. By the action of the bulb the phase of pitching proceeds about $90 \mathrm{deg}$. ahead of the heaving that gives a visual impression of favourable control over the bow motion.

\section{Conclusion}

The most important conclusions drawn from the preceding investigation may be summarised as follows :

(1) The main hull should be a waveless hull form having no parasitic waves. If not attainable practically, the lines should be made as simple and natural as possible.

(2) The location of bulb has a priority order of selection for the waveless effect.

(3) The size of bulb depends on the waveless speed but it should not introduce an increase of the form drag.

(4) The effect of bulb on propulsion is more favourable than expected.

(5) The performance among waves may also be much improved.

(6) The foregoing results may give hopefull suggestions except fears for manoeuvring, anchoring and some operations, but there is a possiblity of the solution if hollow waveless lines are cultivated to bring back the bulb towards FP.

\section{Acknowledgement}

The author wishes to express his gratefulness to Prof. Dr. T. Inui for his continued encouragement and also wishes to thank Prof. Dr. T. Takahei and Dr. M. Kumano for their helpfull supports. The experimental work and the theoretical analysis have all been carried out at the Fishing Boat Laboratory, Fisheries Agency, and thanks are also offered to all members of the tank for their assistance.

\section{Reference}

1) INUI, TAKAHEI, KUMANO : “Tank test of the wave-making effect by bulbous bow" ZKK 108.

2) TAKAHEI : "Study on Waveless Bow" ZKK 108 \& 109.

3) KUMANO : "Study on Waveless Stern" ZKK 108 \& 109.

4) TRAUNG : "The Prismatic Coefficient" FISHING BOATS OF THE WORLD II.

5) YOKOYAMA, KOBAYASHI : “Resistance Test of European Wodden Trawlers" TECHNICAL REPORT OF FISHING BOAT No. 13.

(Abr. ZKK : Journal of “ZOSEN KIOKAI”, The Society of Naval Architects of Japan) 